\title{
Envelope Extraction Algorithm of the Vehicle Acoustic Signal Based on Step-Size Transformation
}

\author{
Zhang-li Lan ${ }^{1, \mathrm{a}}$, Fang Liu ${ }^{1, \mathrm{~b}}$, Wei Chen ${ }^{1, \mathrm{c}}$, Yang Yang ${ }^{1, \mathrm{~d}}$, Fen Huang ${ }^{1, \mathrm{e}}$ \\ ${ }^{1}$ School of Information Science and Engineering, Chongqing Jiaotong University, Chongqing \\ 400074, China

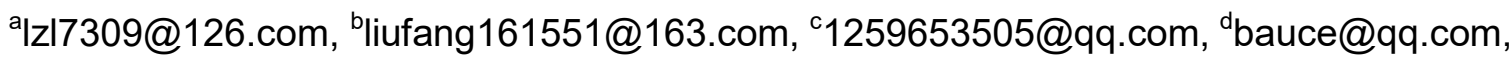 \\ e839553154@qq.com
}

\begin{abstract}
Keywords: intelligent traffic detection; envelope extraction; feature parameter; step-size transformation
\end{abstract}

\begin{abstract}
The waveform of acoustic signal when the vehicle passing the deceleration strip is obviously different from where it driving on the normal road, of which the characteristic parameters can be extracted and is essential to automatically identify the quantity, speed and type of the vehicle. Extracting the characteristic parameters of the acoustic signal envelope has many advantages over the original signal. Combined with the characteristic of the acoustic signal, an acoustic signal envelope extraction algorithm based on step-size transformation for the vehicle when passing the deceleration strip is proposed, which traversing the signal by setting different step-size and drawing curves with the maximum in every step-size to compare with the waveform of original signal so that the envelope can be extracted effectively on the basis of the error value of the contour clarity and feature point extraction. The experimental results show that the proposed algorithm has clearer envelope contour, fewer burrs and smaller error of the characteristic parameters extraction compared to that of the traditional under the same sampling points.
\end{abstract}

\section{Introduction}

With the rapid advancement of the highway traffic, it is very important to master the traffic information that includes the number, type and driving conditions of the vehicle for the traffic management. So, the realization of intelligent, informationalized and high-efficient traffic information detection has become a vital prerequisite for the intelligent traffic management. Acoustic signal analysis-based traffic information detection technology has the advantages of low cost, small information redundancy and small external interference [1-3]. It is obviously different in the waveform of the acoustic signal when the car passing the deceleration belt and driving on the normal highway [4-6]. Meanwhile, for this signal, its envelope curve can better reflect the variation of the feature compared with the original signal. In hence, it plays a significant role in the identification of traffic flow parameters.

Signal envelope extraction is a technique which can find the waveform edge using a certain signal processing method [5]. And the envelope contains some time-domain characteristic parameters which can reflect some properties and features of the signal to some extent. Especially, this technique is of great significance in the traffic detection field like the judgement of the vehicle speed and type. So it is extremely key to adopt a reasonable way to extract the envelope.

Some research has conducted in the envelope extraction field where Hilbert transform method and wavelet transform method have been proposed. The former one is a method to transform the 
time-domain real signal into time-domain analytic signal. The analytic signal contains real part and imaginary part which represents the real signal itself and the Hilbert transform of the real signal respectively, and that the amplitude of the analytic signal is the envelope of the real signal [7-10]. However, the latter method filters low-frequency noise through the high-pass filter firstly, then half-wave rectification, finally hierarchical decomposition for the pre-processing signal and selecting a layer of approximate coefficient of reconstruction as the envelope curve of the original signal [11-14]. Hilbert transform transforms the signal in the whole frequency band, which has no inhibitory effect on the noise of the whole frequency band and has the leakage error at the same time. For the acoustic signal collected in outdoors, the envelope extracted by this method is not smooth enough, has more burr, and it is difficult to truly reflect the nature and characteristics of the signal [12]. Wavelet transform based signal envelope extraction method is usually used in biomedical and fault diagnosis field due to it works well when the signal is weaker, has stronger noise and lower frequency [15].

In this paper, the signal comes from the traffic field has the characteristics of low signal-to-noise ratio (SNR), high sampling frequency and many frequency components, and on the basis of these, a step-size transformation based acoustic envelope extraction algorithm is proposed, which can extract the envelope of the acoustic signal effectively by setting different step and a series of processing (detailed in section 2). Besides, the proposed methodology can lay the foundation for the traffic intelligent detection to some extent.

\section{The proposed algorithm}

Background. Acoustic signal analysis based traffic information detection technology can greatly improve the traffic information detection effect, which can further realize intelligent traffic management. The automatic judgment of the quantity, speed and type of the vehicle is an important part of the traffic detection, which can be realized by the way of analyzing the vehicle sound. Generally, when driving on the normal road, the amplitude of the acoustic signal changes within a certain range and without obvious peak (as shown in Fig. 1). But when the vehicle passing the deceleration belt, human can hear that it sounds like the sound "deng" due to the interaction of wheel and deceleration belt, and it presents the amplitude variation in the form of the signal(as depicted in Fig. 2). It can be seen from the figure that the envelope curve can better reflect the characteristics of this signal than that of the original signal, which contains some characteristic parameters that can reflect the nature and features of the signal to some extent, so it is necessary to study the envelope extraction of this acoustic signal.

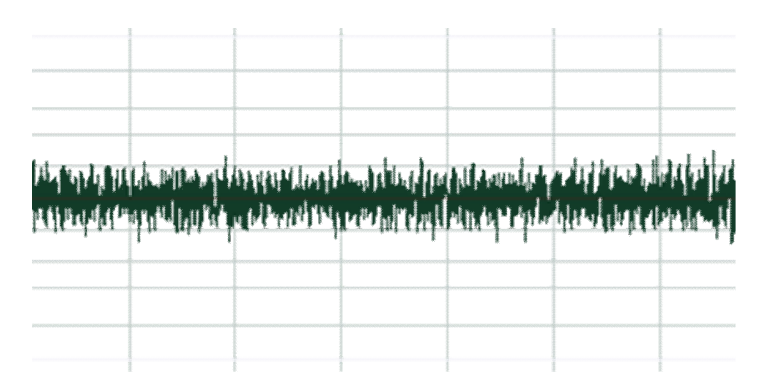

Fig. 1 waveform of the acoustic signal when driving on the normal road

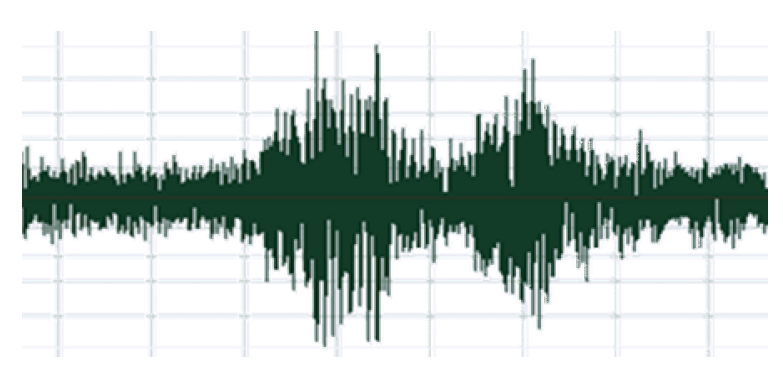

Fig. 2 waveform of the acoustic signal when passing the deceleration belt

In this paper, for this acoustic signal, a step-size transformation-based acoustic signal envelope extraction algorithm is proposed on the basis of the characteristics which is obtained by observing and analyzing the amplified noise reduction signal. Moreover, the envelope extracted effectively by 
the proposed method is advantageous to extract the characteristics of this signal which can be further applied in vehicle identification, vehicle speed detection and traffic statistics.

Description of the proposed algorithm. In the proposed methodology, firstly traversing the waveform of the acoustic signal with the maximum step-size and taking i points in every step-size time, then adopting the method (as shown in Fig. 3) to find the maximum amplitude within the step-size time as well as record the corresponding time value, and then storing them in the corresponding amplitude array and time array respectively. Then drawing the envelope curve with the amplitude array data and the time array data as the vertical and abscissa, respectively. Finally, comparing this curve with the original signal to see if the envelope is ideal, if so, post-processing and then ending; if not, changing the step-size and repeating the operation above until the ideal envelope curve is found.

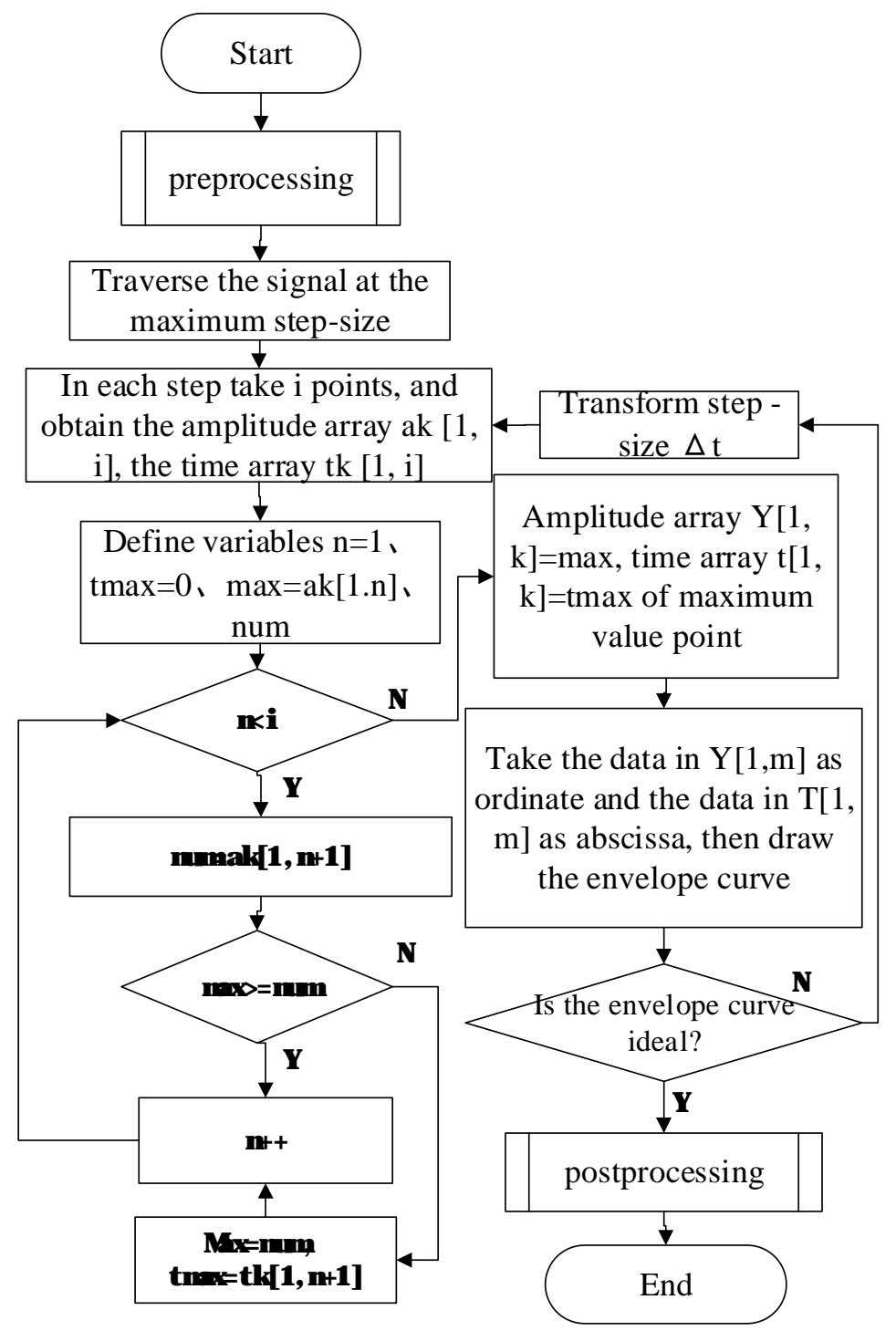

Fig. 3 The flow chart of the algorithm 


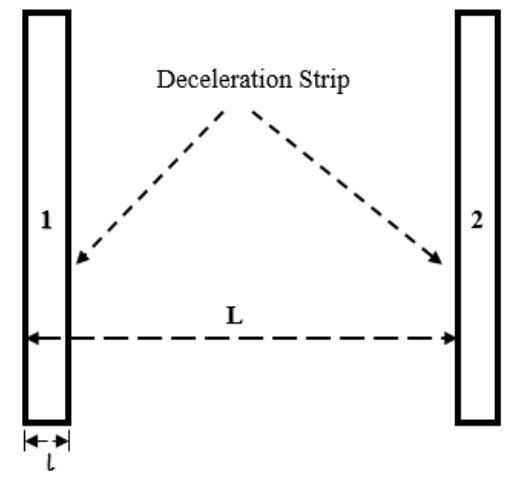

Fig. 4 Schematic diagram of deceleration belt

Where $\mathrm{L}$ is the distance between the two deceleration belts: deceleration belt 1 and deceleration belt $2, l$ is the width of the deceleration belt.

Fig. 4 is the basis of the step-size $\Delta \mathrm{t}$. Suppose the speed is $\mathrm{V}$ and the wheelbase is s. The time which the former row of wheels of the vehicle passing through the deceleration belt 1 is $t 1$, the time which the latter row of wheels of the vehicle passing through the deceleration belt 1 is $t 2$, the time which the former row of wheels of the vehicle passing through the deceleration belt 1 and deceleration belt 2 is $t 3$. Where $t 1, t 2$ and $t 3$ can be calculated by the formula (1), (2) and (3):

$$
\begin{aligned}
& t 1=\frac{l}{v} \\
& t 2=\frac{s}{v} \\
& t 3=\frac{L}{v}
\end{aligned}
$$

(1) If collects the double-crest point when the same row of wheels passing the deceleration belt, the maximum step-size can be adopted by formula (4):

$$
\Delta t \max =\left\lfloor\frac{t 1}{2}\right\rfloor=\left\lfloor\frac{l}{2 v}\right\rfloor
$$

(2) If collects the single-crest point when the same row of wheels passing the deceleration belt, it has two cases:

Case 1: when $L>$ s, the maximum step-size can be adopted by formula (5):

$$
\Delta t \max =\left\lfloor\frac{t 2}{2}\right\rfloor=\left\lfloor\frac{s}{2 v}\right\rfloor
$$

Case 2: when $\mathrm{L}<\mathrm{s}$, the maximum step-size can be adopted by formula (6):

$$
\Delta t \max =\left\lfloor\frac{t 3}{2}\right\rfloor=\left\lfloor\frac{L}{2 v}\right\rfloor
$$


(3) Suppose the signal sampling frequency is $F s$, the time interval between the adjacent sampling points is $\frac{1}{F s}$, then the minimum step-size $\Delta$ tmin can be expressed as follows:

$$
\Delta t \min =\left\lfloor\frac{n}{F_{S}}\right\rfloor \quad n=1,2,3 \mathrm{~L}
$$

Where $\mathrm{n}$ is limited by the selected signal length, and the empirical value can be obtained through experiments, and generally $\mathrm{n}$ take about 10 . The extracted envelope has no differences with the original signal if $\mathrm{n}$ is too small, which will make no sense.

\section{Experiments}

Experiments with the proposed methodology. In the experiments, the distance between the two deceleration strips $\mathrm{L}=25 \mathrm{~m}$, the width of the deceleration strip $l=0.3 \mathrm{~m}$, wheelbase $\mathrm{s}=2.48 \mathrm{~m}$, sampling frequency $\mathrm{Fs}=48000 \mathrm{~Hz}$, signal length $\mathrm{Ts}=2 \mathrm{~S}$.

Collecting the single-crest point when the same row of wheels passing the deceleration belt, according from the basic data above, $\Delta$ tmax belongs to the case 1, that is $\Delta t \max =\left\lfloor\frac{t 2}{2}\right\rfloor=\left\lfloor\frac{s}{2 v}\right\rfloor$.

The vehicle speed is in the range of $40 \mathrm{Km} / \mathrm{h}-100 \mathrm{Km} / \mathrm{h}$ at the data-collected road section, so the range of the maximum step-size $\Delta$ tmax is:

$$
45 m s=\left\lceil\frac{2.48}{2 \times 100 / 3.6}\right\rceil<\Delta t \max <\left\lfloor\frac{2.48}{2 \times 40 / 3.6}\right\rfloor=112 m s
$$

The waveform of the original signal y is shown in Fig. 5:

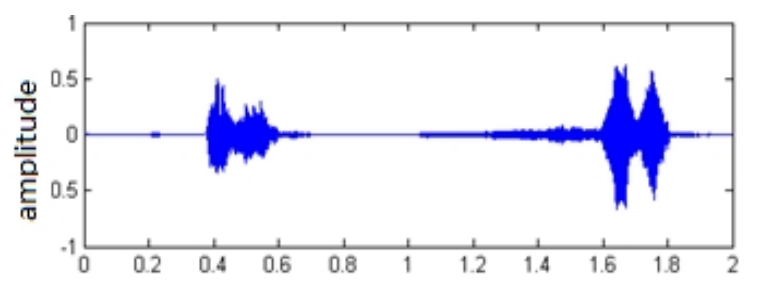

Fig. 5 The waveform of the original signal

The specific experiment steps are as follows:

(1) Take the $\Delta \mathrm{t}=80 \mathrm{~ms}$ as the initial step-size and traverse signal y:

1) Select 3840 points in every $\Delta \mathrm{t}$ time, then put the amplitude data into ak[1,i] and put the time data into tk[1, i];

2) Find the maximum in $a k[1, i]$ and record the corresponding time data, then store them in the amplitude array $\mathrm{Y}[1,25]$ and the time array $\mathrm{T}[1,25]$ respectively;

(2) Take the data in $\mathrm{Y}[1,25]$ as ordinate and the data in $\mathrm{T}[1,25]$ as abscissa, then draw the envelope curve as shown in Fig. 6;

(3) Compared to the original signal waveform, finding Fig. 6 is not the ideal envelope;

(4) Change the step-size $\Delta \mathrm{t}$ to $\Delta \mathrm{t}=40 \mathrm{~ms}$ and traverse signal $\mathrm{y}$ :

1) Select 1920 points in every $\Delta \mathrm{t}$ time, then put the amplitude data into ak[1,i] and put the time data into $\mathrm{tk}[1, \mathrm{i}]$;

2) Find the maximum in $a k[1, i]$ and record the corresponding time data, then store them in 
the amplitude array $\mathrm{Y}[1,50]$ and the time array $\mathrm{T}[1,50]$ respectively;

(5) Take the data in $\mathrm{Y}[1,50]$ as ordinate and the data in $\mathrm{T}[1,50]$ as abscissa, then draw the envelope curve as shown in Fig. 7;

(6) Adopt the similar process above when changing the step-size $\Delta \mathrm{t}$ to $\Delta \mathrm{t}=20 \mathrm{~ms}, 10 \mathrm{~ms}, 8 \mathrm{~ms}$ and $2 \mathrm{~ms}$;

(7) The result are shown in Fig. 8, Fig. 9 and Fig. 10 respectively.

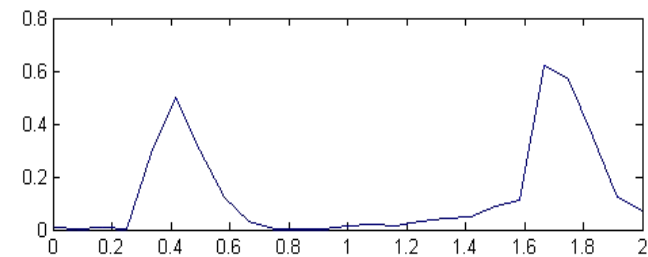

Fig. 6 the envelope curve when $\Delta \mathrm{t}=80 \mathrm{~ms}$

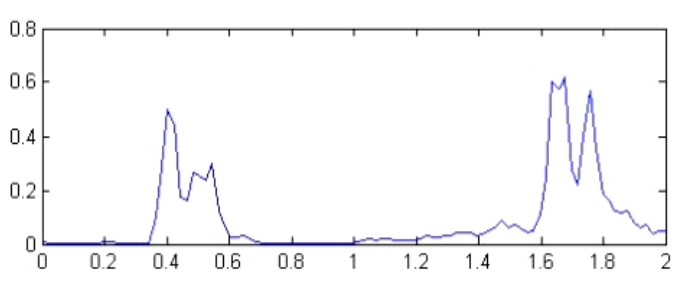

Fig. 8 the envelope curve when $\Delta t=20 \mathrm{~ms}$ Fig. 9 the envelope curve when $\Delta t=10 \mathrm{~ms}$

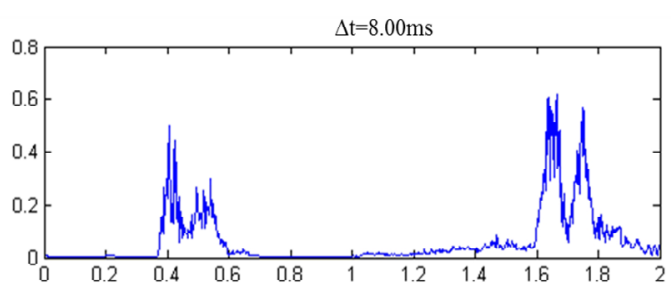

(a)

Fig. 10 (a) the envelope curve when $\Delta \mathrm{t}=8 \mathrm{~ms}$

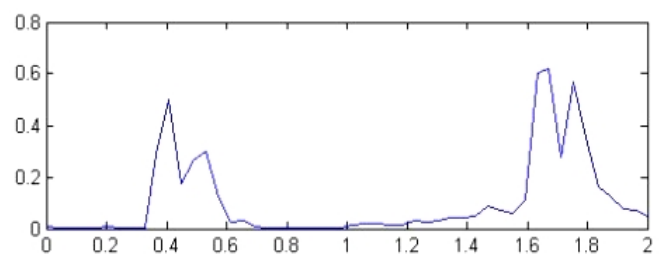

Fig. 7 the envelope curve when $\Delta \mathrm{t}=40 \mathrm{~ms}$
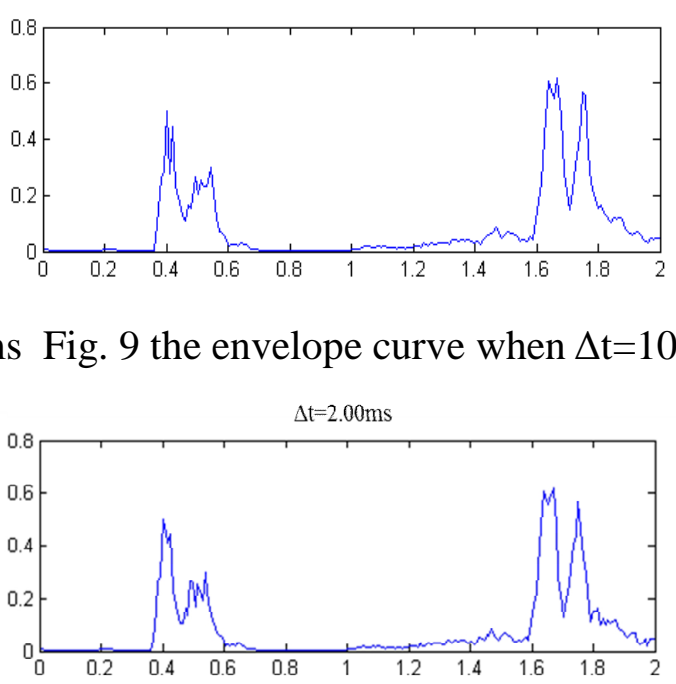

(b)

Comparison experiment. For the same acoustic signal with the same selected points, using wavelet transform method and Hilbert transform method to extract envelope respectively and the results are as follows:
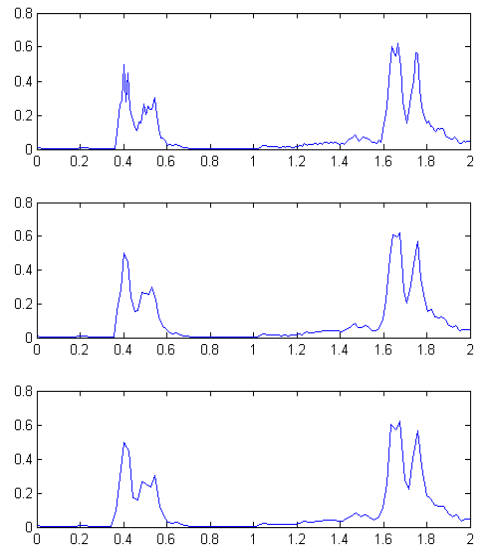

Fig. 11 the envelope curve extracted by the proposed method under different resampling frequencies
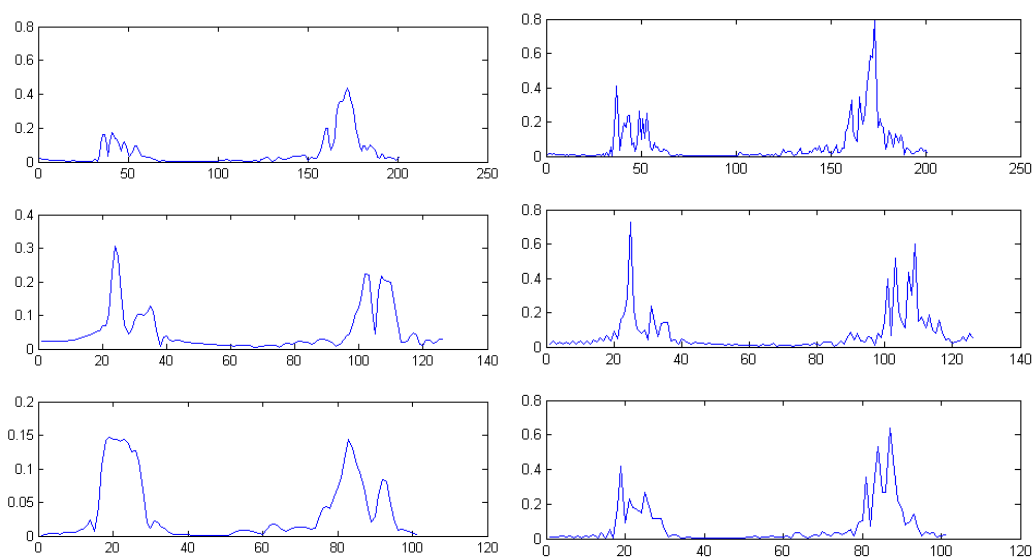

Fig. 12 the envelope curve extracted by wavelet transform method under different resampling frequencies

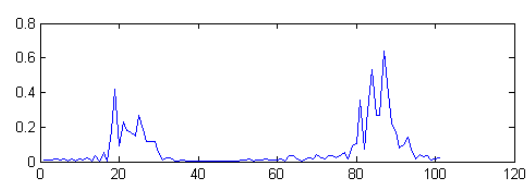

Fig. 13 the envelope curve extracted by Hilbert transform method under different resampling frequencies 
Analysis. As can be seen from the experiment in section 3.1, using different step-size to traverse and sample the original signal can obtain corresponding envelope curve. When $\Delta \mathrm{t}<10 \mathrm{~ms}$, the smaller the $\Delta \mathrm{t}$, the more burr of the extracted envelope; when $10 \mathrm{~ms}<=\Delta \mathrm{t}<=20 \mathrm{~ms}$, with the increase of $\Delta \mathrm{t}$, the envelope curve is clearer and the burr is fewer, and the characteristic becomes more obvious, so this step-size area is determined to be the ideal area; when $\Delta \mathrm{t}>20 \mathrm{~ms}$, the peak point of the envelope curve becomes blurred and the feature point is lost with the increase of $\Delta t$. Due to the envelope curve is obvious when $10 \mathrm{~ms}<=\Delta \mathrm{t}<=20 \mathrm{~ms}$, so extract the characteristic point of the crest when $\Delta \mathrm{t}=10 \mathrm{~ms} 、 16 \mathrm{~ms} 、 20 \mathrm{~ms}$, and considering that the deceleration can cause a minimum interval peak in a crest, the first crest is adopted as the peak extracted point, the result of the crest time extraction is shown in the table 1 .

Table 1 the crest time extraction of the proposed algorithm

\begin{tabular}{ccccc}
\hline \multirow{2}{*}{$\begin{array}{c}\text { Step-size } \Delta \mathrm{t} \\
(\mathrm{ms})\end{array}$} & $\begin{array}{c}\text { The first crest } \\
\text { corresponding to } \\
\text { the former wheel }\end{array}$ & $\begin{array}{c}\text { The first crest } \\
\text { corresponding to } \\
\text { the latter wheel }\end{array}$ & $\begin{array}{c}\text { The second crest } \\
\text { corresponding to } \\
\text { the former wheel }\end{array}$ & $\begin{array}{c}\text { The second crest } \\
\text { corresponding to } \\
\text { the former wheel }\end{array}$ \\
\cline { 2 - 5 } 10 & 0.4020 & 0.4925 & 1.6382 & 1.7487 \\
16 & 0.4032 & 0.4839 & 1.6451 & 1.7580 \\
20 & 0.4040 & 0.4848 & 1.6363 & 1.7576 \\
\hline
\end{tabular}

A great deal of practice has proved that the existence of error is inevitable and universal, but study the existed error is beneficial to reduce even eliminate the error. Since the true value is an ideal concept and it is generally unknown. But in some specific cases, the observed value under the specific conditions can be viewed as the true value. In this experiment, video is recorded at the same time when collecting the acoustic signal, and using specific image observation software to extract the time when the vehicle rolling the deceleration belt, which is viewed as this experiment true value. As shown in table 2 .

Table 2 The actual value of the peak time point of the original signal

\begin{tabular}{|c|c|c|c|c|}
\hline \multirow{3}{*}{$\begin{array}{l}\text { under the } \\
\text { specific } \\
\text { conditions }\end{array}$} & $\begin{array}{l}\text { The first crest } \\
\text { corresponding to } \\
\text { the former wheel }\end{array}$ & $\begin{array}{l}\text { The first crest } \\
\text { corresponding to } \\
\text { the latter wheel }\end{array}$ & $\begin{array}{l}\text { The second crest } \\
\text { corresponding to } \\
\text { the former wheel }\end{array}$ & $\begin{array}{l}\text { The second crest } \\
\text { corresponding to } \\
\text { the former wheel }\end{array}$ \\
\hline & \multicolumn{4}{|c|}{$(\mathrm{s})$} \\
\hline & 0.4051 & 0.4945 & 1.6380 & 1.7514 \\
\hline
\end{tabular}

Combined the table 1 and table 2, the error result is shown in table 3. As can be seen from the table3, within the valid step-size, the relative error range is $0.01 \%-2.36 \%$, the average error range is $0.33 \%-1.24 \%$, and the error variance range is $0.52 \%-3.64 \%$. Besides, under the three step-size, the average error and error variance are minimum when $\Delta \mathrm{t}=10.00 \mathrm{~ms}$, which is the most suitable time to extract the envelope curve so as to accord with the changing rule and characteristics of the original signal, as a result, the extracted envelope curve under this step-size can be served as that of the original signal. 
Table 3 the result of the error analysis

\begin{tabular}{|c|c|c|c|c|c|c|}
\hline \multirow{2}{*}{$\begin{array}{l}\text { Step-size } \Delta \mathrm{t} \\
(\mathrm{ms})\end{array}$} & \multicolumn{4}{|c|}{$\delta$} & $\bar{\delta}$ & $D$ \\
\hline & \multicolumn{6}{|c|}{$\%$} \\
\hline 10 & -0.77 & -0.40 & -0.01 & -0.15 & 0.33 & 0.52 \\
\hline 16 & -0.47 & -2.14 & 0.43 & 0.38 & 0.86 & 2.80 \\
\hline 20 & -0.27 & -1.96 & 2.36 & 0.35 & 1.24 & 3.64 \\
\hline
\end{tabular}

Where $\delta$ is relative error, $\bar{\delta}$ is average error, and $D$ is error variance.

As for the comparison experiment in section 3.2, it can be found that the envelope extracted by the Wavelet transform has fewer burrs and the contour is clearer than that of the Hilbert transform with the change of the resampling frequency. Furthermore, it is obvious that the smaller the resampling frequency is, the contour of the envelope curve is clearer while the feature points are lost more seriously. Moreover, the extracted crest time of the envelope curve with the three methods under the same sampling frequency is shown in table4, and the errors of the three methods is shown in table 5 .

Table 4 the extracted crest time of the envelope curve with the three methods under the same sampling frequency

\begin{tabular}{|c|c|c|c|c|}
\hline \multirow[t]{2}{*}{ method } & $\begin{array}{c}\text { The first crest } \\
\text { corresponding } \\
\text { to the former } \\
\text { wheel }\end{array}$ & $\begin{array}{c}\text { The first crest } \\
\text { corresponding } \\
\text { to the latter } \\
\text { wheel }\end{array}$ & $\begin{array}{l}\text { The second crest } \\
\text { corresponding to } \\
\text { the former wheel }\end{array}$ & $\begin{array}{l}\text { The second crest } \\
\text { corresponding to } \\
\text { the former wheel }\end{array}$ \\
\hline & \multicolumn{4}{|c|}{$(\mathrm{S})$} \\
\hline $\begin{array}{c}\text { The proposed } \\
\text { method }\end{array}$ & 0.4020 & 0.4925 & 1.6382 & 1.7487 \\
\hline Wavelet transfor & 0.4050 & 0.5550 & 1.6450 & 1.7150 \\
\hline Hilbert transfor & 0.4225 & 0.4885 & 1.6351 & 1.7250 \\
\hline
\end{tabular}

Table 5 the errors comparison of the three methods

\begin{tabular}{ccccccc}
\hline \multirow{2}{*}{ method } & \multicolumn{3}{c}{$\delta$} & $\delta$ & $D$ \\
\cline { 2 - 6 } & \multicolumn{7}{c}{$\%$} & & \\
\hline $\begin{array}{c}\text { The proposed } \\
\text { method }\end{array}$ & -0.77 & -0.40 & 0.01 & -0.15 & 0.33 & 0.52 \\
Wavelet transform & -0.02 & 12.23 & 0.43 & -2.08 & 3.69 & 32.67 \\
Hilbert transform & 4.30 & -1.21 & -0.18 & -1.51 & 1.80 & 7.55 \\
\hline
\end{tabular}

Where $\delta$ is relative error, $\delta$ is average error, and $D$ is error variance.

From the table 4 and table 5, it can be found that the feature points of envelope curve obtained by wavelet transform and Hilbert transform methods are seriously lost when at the same resampling frequency. In these three methods, the relative error range of the proposed method is the smallest, 
the Hilbert transform method comes second while the wavelet transform is the last. Besides, the average error and the error variance of the proposed algorithm are all smaller than that of the other two methods.

\section{Conclusions}

From the experiments and comparison, it can be known that the step-size transformation-based acoustic signal envelope extraction algorithm for vehicle passing the deceleration strip can realize the envelope extraction of acoustic signal based on contour definition and feature point extraction error. For the acoustic signal applied in this paper, the errors of the extracted feature points are small, such as the relative error range is $0.01 \%-2.14 \%$, the average error range is $0.38 \%-0.94 \%$, and the error variance range is $0.59 \%-3.11 \%$. What's more, the experiments has verified that the feasibility to the ideal envelope extraction which based on the step-size $\Delta \mathrm{t}$ that in the light of the contour definition and feature point extraction error. Generally, the proposed algorithm can realize the extraction of the characteristic parameters which makes for achieving the automatic judgment of the number, speed and type of vehicle in the traffic field.

\section{Acknowledgements}

This work was financially supported by Chongqing Research Projects for Basic Science and Advanced Technology (cstc2016jcyjA1953).

\section{Reference}

[1] Shun Jin. Vehicle Identification Research Based on Feature Fusion of Acoustic and Seismic Signals[D]. Xi'an: Chang'an University, 2014.

[2] Ming-yuan Bian, Si-zhong Chen, Han-jun Luo, et al. Development and Prospect of Intelligent Transportation Systems[J]. Journal of Wuhan University of Technology Information and Management Engineering Edition, 2001, 23 (1): 67-70.

[3] Yun-huan Li. Research of Traffic Information Detection Technology Based on Voice Recognition[D]. Xi'an: Chang'an University, 2014.

[4] Ji-qing Han, Ran Tie. Speech signal processing[M]. Beijing: Tsinghua University Press, 2004.

[5] Zhi Li, Xiang-chu Chen, Zheng-bo Liu. Envelope Analysis and Its Application in Equipment Fault Diagnosis[J]. Journal of Test and Measurement Technology, 2002, 16 (2): 92-95.

[6] Guo-juan Cai. Bearing Fault Diagnosis Based on Envelope Analysis[J]. Petrochemical Equipment Technology, 2014, 35(2):41-43.

[7] Guang-rong Wang. Research on Signal Envelope Extraction Method Based on Hilbert Transform[J]. China Science and Technology Information, 2012 (1): 87-88

[8] Xu-sheng Zhang, Yi-sheng Zhu, Xiao-xiong Cheng, et al. Signal envelope extraction methodfrom Hilbert transform to wavelet transform[J]. Electronic Science, 1997, 19 (1): 120-123 .

[9] Huang D. A Wavelet-Based Algorithm For The Hilbert Transform[J]. Mechanical Systems \& Signal Processing, 1996, 10(2):125-134.

[10] Ying-ying Zhang, Hong-xia Pan, Mao-yuan Zheng. Fault Diagnosis of Rolling Bearings Based on Wavelet Packet and Hilbert Envelope Analysis[J]. Electronic Testing, 2010 (6): 20-23. 
[11]Jian-cang Ma, Lei Luo. Envelope Analysis Based on Wavelet Transform[J]. Journal of Northwestern Polytechnical University, 1997 (2): 249-253.

[12]Bin Liu, Gui-ping Dai, Hong-bin Lin. An Improved Algorithm for Envelope Extraction Based on Wavelet Transform[J]. Journal of Instrumentation, 2006, 27 (1): 34-37.

[13] Hua-juan Yang, Xi-qiang Qu, Yan Han. Enhancement of acoustic signal envelope based on wavelet transform[J]. Journal of North University of China (Natural Science Edition), 2004,25 (4): 300-302.

[14] Tie-shuang Hou. Signal envelope detection based on complex analytic wavelet transform[J]. Journal of Xi'an University of Posts and Telecommunications, 2011, 16 (3): 18-21.

[15]Su Zhou, Di Zhu, Xiao-ming Wu, et al. Envelope extraction algorithm and phonocardiogram signal application based on wavelet transform[J]. China Tissue Engineering Research,2011,15 (30): 5615-5619. 